Claremont Colleges

Scholarship@ Claremont

All HMC Faculty Publications and Research

HMC Faculty Scholarship

$1-1-1994$

\title{
A Theoretical Investigation of the Stability of HPC
}

Hannah S. Hong

Harvey Mudd College

Robert J. Cave

Harvey Mudd College

\section{Recommended Citation}

Hong, H.S. ;Cave, R.J. “A Theoretical Investigation of the Stability of HPC,” J. Phys. Chem. 1994, 98, 10036. DOI: 10.1021/ j100091a016

This Article is brought to you for free and open access by the HMC Faculty Scholarship at Scholarship @ Claremont. It has been accepted for inclusion in All HMC Faculty Publications and Research by an authorized administrator of Scholarship @ Claremont. For more information, please contact scholarship@cuc.claremont.edu. 


\title{
A Theoretical Investigation of the Stability of HPC
}

\author{
Hannah S. Hong and Robert J. Cave, \\ Department of Chemistry, Harvey Mudd College, Claremont, California 91711 \\ Received: February 2, 1994; In Final Form: June 28, $1994^{\otimes}$
}

\begin{abstract}
Several $a b$ initio methods are applied to the study of two linear isomers of methylidyne phosphine, HCP and HPC. Single-reference correlation methods ranging from second-order Møller-Plesset theory to coupled cluster singles and doubles theory with noniterative inclusion of connected triple excitations were applied in a variety of basis sets of increasing size. In addition, complete active space self-consistent field wave functions, multireference singles and doubles configuration interaction, and averaged coupled pair functional theory were also applied to HPC. For HCP comparison of the single-reference based results is made with experimental data and previous theory to assess the accuracy of the methods employed. The same single-reference-based methods are then applied to linear HPC in order to assess whether it is a stable minimum or a transition state (imaginary frequencies in two dimensions). It is found that linear HPC is not a minimum on the groundstate potential surface. However, relatively high levels of correlation must be used when single-referencebased methods are employed to arrive at this conclusion. The multireference-based methods are then applied to HPC, and they too predict that it is not a minimum on the ground electronic potential surface. The qualitative nature of linear HPC is examined using CASSCF and CCSD wave functions.
\end{abstract}

\section{Introduction}

Hydrogen cyanide is a system which has received a great deal of theoretical and experimental attention recently. ${ }^{1-6}$ Much of this work has focused on the ground-state surface, which is made particularly interesting by the presence of two stable isomers, linear HCN (the lowest energy configuration) and $\mathrm{HNC}$ (approximately $0.64 \mathrm{eV}$ above $\mathrm{HCN}^{1}$ ). Since this system is well studied experimentally, ${ }^{5,6}$ the possibility exists of doing highquality calculations of vibrational spectra on an accurate ab initio surface to yield theoretical spectra for comparison with experiment.

Experimental studies have also examined vibrational motion on the ground-state surface of HCP, a substituted analogue of $\mathrm{HCN} .^{7-10}$ It is known that the ground-state global minimum is linear and of the form $\mathrm{H}-\mathrm{C}-\mathrm{P} ;{ }^{11}$ experimentally it is not known whether the isomer $\mathrm{H}-\mathrm{P}-\mathrm{C}$ exists. It is known from stimulated emission pumping experiments ${ }^{8}$ that the ground-state bending potential is more harmonic than was expected based on previous theory ${ }^{7}$ and that highly excited bending states can be accessed with little CP stretch being induced. One cannot yet infer from this data whether linear HPC exists, as the states studied were not high enough in energy to probe this portion of the potential surface (up to $54 \mathrm{kcal} / \mathrm{mol}$ above the HCP minimum, whereas HPC is calculated to be about $80 \mathrm{kcal} / \mathrm{mol}$ above HCP; see refs 7 and 12, and below).

Theoretically, HCP has been studied using a variety of methods and basis sets. ${ }^{7 a, c, 12-20}$ The earlierst series of calculations used relatively small basis sets and modest correlation methods, ${ }^{13-18}$ but the first calculations to address the stability of HPC were performed by Lehmann et al. ${ }^{\text {7a }}$ They used frozenangle restricted Hartree-Fock geometries followed by fourthorder Møller-Plesset (singles, doubles, and quadruples) to construct a bending potential for HCP, and predicted that HPC is not a stable species. The density of points near linear HPC was probably not sufficient to detect a small energy rise as the molecule departed from linearity, but their study has proven to be a quite useful point of comparison for later theory and

\footnotetext{
* Author to whom correspondence should be sent.

† Camille and Henry Dreyfus Teacher-Scholar, 1993-1998.

Q Abstract published in Advance ACS Abstracts, August 15, 1994
}

experiment. Karna et al. ${ }^{19}$ used multireference singles and doubles configuration interaction wave functions to examine the ground and certain excited states of HCP in a relatively large basis set. They suggested assignments for several excited states, obtained vibrational frequencies for several states, and calculated bending potentials at fixed bond lengths, but did not move far enough around in the bending coordinate to examine the HPC isomer. More recently, Bloor and $\mathrm{Yu}^{20}$ examined the sensitivity of the geometry and many one-electron properties of HCP to the choice of basis set and correlation treatment. Their work emphasized the importance of extended basis sets and extensions of the correlation treatment beyond second-order Møller-Plesset perturbation theory (MP2) to achieve accurate geometries and properties. They too focused on the HCP isomer.

The most recent work to address the question of the stability of HPC is that of Goldstein et al. ${ }^{12}$ They used up to triple zeta polarization (TZP) basis sets with either MP2 or CASSCF (complete active space self-consistent field) wave functions to examine both HCP and HPC. They concluded that the potential surface was flat in the region of HPC but the two correlation methods gave conflicting predictions as to whether HPC was a minimum (MP2, minimum; CASSCF, 2D saddle point). RHF results in all basis sets predicted that HPC was not a minimum. The CASSCF treatment (at most 10 electrons in 10 orbitals) gave excellent agreement with experiment in the case of $\mathrm{HCP}$ vibrational frequencies, but it was not clear whether its limited correlation recovery was yielding incorrect predictions for HPC. On the other hand, it could also be argued that the singlereference nature of the MP2 was inaccurate for the triply bonded system, or that higher order perturbation effects could be important in this system. Thus while both methods led to the conclusion that the surface is flat near linear HPC, the question of whether HPC is a true minimum was left open.

With these questions in mind, we undertook the present investigation. We used significantly larger basis sets than have been applied before to HPC, and single-reference correlation methods up to the coupled-cluster singles and doubles with noniterative inclusion of connected triples model ${ }^{21}(\operatorname{CCSD}(\mathrm{T}))$. We have also applied several multireference-based correlation methods to HPC. It is found that it is critical to go beyond 
MP2 for HPC vibrational frequencies, as the bending frequency oscillates from imaginary to real for low orders of perturbation theory. We also find that while linear HCP appears to be well described by a single-reference-based correlation model, there are indications that there are significantly greater nondynamical correlation effects near the minimum for HPC, based on results of calculations of the $T_{1}$ diagnostic of Lee et al. ${ }^{22}$ (The CASSCF description indicates the difference arises due to greater excitation out of a $\sigma$ orbital for HPC.) On the basis of the present results we predict that HPC is not a stable isomer of methylidyne phosphene.

In what follows, we examine both basis set and correlation treatment effects on the geometries and vibrational frequencies for HCP and HPC. In general it is found that a triple zeta plus polarization basis (TZP) is sufficient to yield a semiquantitative description of HPC, but that if single-reference methods are employed, high levels of correlation treatment are required to obtain converged results. In section II, the basis sets and correlation methods that were used are outlined, followed by a presentation of the results in section III. In section IV we discuss the results, and section $\mathrm{V}$ contains a brief summary of our conclusions.

\section{Theoretical Methods}

All restricted Hartree-Fock (RHF) and Moller-Plesset (MP) perturbation theory calculations were performed using the Gaussian 92 (G92) suite of electronic structure codes. ${ }^{23}$ Most of the coupled cluster results (both coupled cluster singles and doubles (CCSD) and coupled cluster singles and doubles with noniterative inclusion of connected triples $\left(\operatorname{CCSD}(T)^{21}\right)$ were performed with G92 as well, except that some single-point calculations were performed with $\mathrm{PSI}^{24}$ in order to calculate the $T_{1}$ diagnostic. ${ }^{22}$ The $T_{1}$ diagnostic has been proposed as a measure of the degree of nondynamical correlation effects in the $\mathrm{CC}$ wavefunction, and hence an indicator of the reliability of the single-reference description for the case under study. ${ }^{22}$ The majority of the CASSCF calculations, and all multireference singles and doubles configuration interaction (MRSDCI) and averaged coupled pair functional ${ }^{25}$ (ACPF) theory calculations, were performed using MOLCAS. ${ }^{26}$ The MRSDCI and MRACPF calculations were performed using the interacting space option: double excitations from inactive to virtual orbitals were not allowed that would have the inactive and virtual electrons couple to a triplet.

All correlated calculations treat only the valence electrons, and only the valence electrons are correlated in the vibrational frequency calculations. In cases where the bending frequencies are not exactly degenerate due to numerical differentiation errors we have taken the average of the two values and quoted the average in the tables. In the multireference cases, the version of MOLCAS we used did not possess analytical gradients. We performed preliminary calculations on linear HPC using MRSDCI, MRSDCI+Q (+Davidson correction for quadruple excitations) and MRACPF, fitting the points to a quadratic in the two bond stretches plus a coupling term between the two stretches and found that the minima for the various methods were with $0.01 \AA$ of those obtained by $\operatorname{CCSD}(\mathrm{T})$ in the $6-31 \mathrm{G}^{* *}$ basis. Thus, in all cases reported below we have frozen the $R_{\mathrm{HP}}$ and $R_{\mathrm{CP}}$ bond lengths at 1.4213 and $1.62918 \AA$, respectively, these values being the $\operatorname{CCSD}(\mathrm{T})$ values in the $6-31 \mathrm{G}^{* *}$ basis. While geometry changes may have some effect on the computed force constants, they are expected to be modest over the geometry variations one might see as a function of basis set. The force constants obtained were obtained from a least-squares fit to a simple quadratic in bending angle, based on calculations at $180^{\circ}$,
TABLE 1: HCP Results in 6-311G** Basis

\begin{tabular}{lcccccc}
\hline \multicolumn{1}{c}{ method } & $\begin{array}{c}E(\mathrm{au}) \\
+379.0\end{array}$ & $\begin{array}{c}R_{\mathrm{CH}} \\
(\AA)\end{array}$ & $\begin{array}{c}R_{\mathrm{CP}} \\
(\AA)\end{array}$ & $\omega_{1}, \omega_{2}$ & $\omega_{3}$ & $\omega_{4}$ \\
\hline RHF & -0.13836 & 1.064 & 1.514 & 818 & 1471 & 3518 \\
MP2 & -0.39556 & 1.076 & 1.559 & 683 & 1259 & 3359 \\
MP4(SDTQ) & -0.42687 & 1.080 & 1.567 & 663 & 1220 & 3392 \\
CCSD & -0.40873 & 1.076 & 1.547 & 692 & 1326 & 3363 \\
CCSD(T) & -0.42401 & 1.077 & 1.555 & 661 & 1289 & 3342 \\
Expt. $^{21}$ & - & 1.069 & 1.540 & 675 & 1278 & 3217
\end{tabular}

${ }^{a}$ The theoretical frequencies are harmonic frequencies, the experimental values are fundamental frequencies; both are in $\mathrm{cm}^{-1}$.

$179^{\circ}$, and $178^{\circ}$. In the tables we quote bending frequencies, calculated at the rigid geometry, based on the fit force constant, using an expression from Herzberg. ${ }^{27}$

In cases where the Davidson correction ${ }^{28}$ for quadruple excitations was used the correction was calculated as $\Delta E_{\text {SDCI }}\left(\left(1-\sum c_{01}{ }^{2}\right) / \sum c_{0 i}{ }^{2}\right)$, where $\sum c_{0 i}{ }^{2}$ is the sum of the squares of the reference configurations in the final $\mathrm{CI}$ wave function.

We performed a variety of CASSCF calculations, denoted as $n / m$ CASSCF, where $n$ denotes the number of electrons correlated and $m$ denotes the number of orbitals used. In all CASSCFs we correlated at least the $4 \pi$ electrons and $2 \sigma$ electrons in $4 \pi$ and $2 \sigma$ orbitals (6/6 CASSCF). In the $10 / 10$ CASSCFs four $\sigma$ electrons and $4 \sigma$ orbitals were added to the $6 / 6$ CAS respectively. The $10 / 12$ CASSCF adds two more $\pi$ orbitals to the 10/10 CASSCF.

For the single-reference methods all basis sets used were built upon the $6-31 \mathrm{G}^{29}$ or $6-311 \mathrm{G}^{30}$ basis sets. Note that for $\mathrm{P}$ the " $6-311 \mathrm{G}$ " basis is the triple zeta basis due to McLean and Chandler. ${ }^{31}$ The polarization and diffuse function sets were also those internal to Gaussian $92 .{ }^{32,33}$ In the results presented in the $6-31 \mathrm{G}^{* *}$ basis, only the five $l=2$ components of the six Cartesian $d$ functions are included in the calculation. In the 6-311G basis sets all six Cartesian d functions but only the seven $l=3$ Cartesian f-type functions were used. In test calculations we have seen that the inclusion or exclusion of the lower $l$ components of the Cartesian d functions has essentially no effect on the results. In the multireference cases we used the averaged natural orbital (ANO) basis sets of Widmark et al. ${ }^{34}$ The lower angular momentum contaminants were not deleted for the ANO basis sets. The specific basis sets used are given in the Results section.

In order to investigate the qualitative nature of HCP and HPC near their respective linear stationary points we have performed 10/10 CASSCFs in the 6-31G** basis (six Cartesian d functions) using GAMESS. ${ }^{35}$ Natural orbital occupations were calculated based on the converged results and are discussed below.

\section{Results}

(a) Single-Reference-Based Methods. We first present results for HCP using various basis sets and wave functions in order to assess the quality of the methods to be applied to HPC. Table 1 contains a series of results in the $6-311 \mathrm{G}^{* *}$ basis for HCP. Included are total energies, geometries, and vibrational frequencies. It is seen that there is little change in the geometry or vibrational frequencies in this basis once one goes beyond the Hartree-Fock level of approximation. The frequencies are quite good for the bend and $\mathrm{CP}$ stretch; the $\mathrm{CH}$ stretch is high by about $100 \mathrm{~cm}^{-1}$. Both bond lengths are long by about 0.01 $\AA$ at the $\operatorname{CCSD}(\mathrm{T})$ level, and it is also seen that inclusion of triple excitations has a significant effect on both the CP bond length and the $\mathrm{CP}$ stretching frequency. This is to be expected since in HCP the CP bond is a triple bond, and single-reference methods tend to require relatively high levels of excitations in order to properly describe multiple bonding. However, we have 
TABLE 2: MP2 Results for HCP vs Basis Set

\begin{tabular}{lcccccc}
\hline \multicolumn{1}{c}{ basis } & $\begin{array}{c}E(\mathrm{au}) \\
+379.0\end{array}$ & $\begin{array}{c}R_{\mathrm{CH}} \\
(\AA)\end{array}$ & $\begin{array}{c}R_{\mathrm{CP}} \\
(\AA)\end{array}$ & $\omega_{1}, \omega_{2}$ & $\omega_{3}$ & $\omega_{4}$ \\
\hline $6-311 \mathrm{G}^{* *}$ & -0.3956 & 1.076 & 1.559 & 683 & 1259 & 3359 \\
6-311++G** & -0.3969 & 1.077 & 1.559 & 672 & 1258 & 3356 \\
$6-311 \mathrm{G}(2 \mathrm{~d}, 2 \mathrm{p})$ & -0.4159 & 1.070 & 1.558 & 606 & 1232 & 3356 \\
$6-311 \mathrm{G}(2 \mathrm{df}, 2 \mathrm{pd})$ & -0.4458 & 1.071 & 1.553 & 707 & 1259 & 3372 \\
$6-311 \mathrm{G}(3 \mathrm{df}, 3 \mathrm{pd})$ & -0.4506 & 1.072 & 1.553 & 693 & 1259 & 3340 \\
Expt. $^{21}$ & & 1.069 & 1.540 & 675 & 1278 & 3217
\end{tabular}

${ }^{a}$ The theoretical frequencies are harmonic frequencies, the experimental values are fundamental frequencies; both are in $\mathrm{cm}^{-1}$

computed the $T_{1}$ diagnostic in the $6-31 \mathrm{G}^{* *}$ basis at essentially the minimum for the $\operatorname{CCSD}(\mathrm{T})$ result of Table 1 and found a value of 0.017 , indicating that the single-reference description provided by CCSD or CCSD(T) should be quite reliable for HCP near its minimum. ${ }^{22.36}$ The correlated results in this basis are quite similar to those obtained in our previous work ${ }^{12}$ using a 10/10 CASSCF wave function in a triple zeta polarized basis, except that the CASSCF bond lengths are another $0.01 \AA$ too long, and the $\mathrm{CH}$ stretching frequency is about $50 \mathrm{~cm}^{-1}$ lower for the CASSCF result. This lower frequency for the CASSCF may be due to a cancellation of errors in force constants and geometries, ${ }^{37}$ or could arise from the limited correlation treatment, and so should not be taken as necessarily being more accurate for "the right reasons".

In comparisons of experimental and theoretical vibrational frequencies it is important to note that the experimental values quoted are fundamental frequencies, whereas the theoretical values are harmonic frequencies. (We associate the mode with frequency near $1300 \mathrm{~cm}^{-1}$ with the $\mathrm{CP}$ stretch and the 3400 (HCP) or 2300 (HPC) $\mathrm{cm}^{-1}$ modes with the HX stretches. The degenerate mode is the bending frequency.) In studies of $\mathrm{HCN}^{1,38}$ it is seen that the fundamental frequency for the $\mathrm{H}-\mathrm{C}$ stretch is significantly lower $\left(100 \mathrm{~cm}^{-1}\right)$ than the experimental or theoretical harmonic values. The other two modes are somewhat less affected. It does not appear that enough data has been gathered for the two stretching modes of HCP to obtain accurate harmonic frequencies, ${ }^{9}$ but for the bend the harmonic value is $687.9 \mathrm{~cm}^{-1}$, as opposed to the fundamental value of $674.7 \mathrm{~cm}^{-1} .9$ A simple fit to the first two vibrational transitions involving the ground state for each of the stretching modes yields values of $3273 \mathrm{~cm}^{-1}$ for the HC stretch and $1284 \mathrm{~cm}^{-1}$ for the CP stretch; each in somewhat better agreement with the correlated results of Table 1 (and later results), especially for the $\mathrm{HC}$ stretch.

We have examined HCP in larger basis sets $\left(6-311++\mathrm{G}^{* *}\right.$ to 6-311G(3df,3pd)) using RHF and MP2 wave functions (Table 2). We find that diffuse functions have little effect on the geometry or vibrational frequencies, but there are sizable effects upon inclusion of a second set of first polarization $(\mathrm{d}, \mathrm{p})$ and upon inclusion of a first set of second polarization functions (f,d) (added sequentially). In the MP2 case the second set of (d,p) functions leads to a significant drop in the bending frequency $\left(75 \mathrm{~cm}^{-1}\right)$ followed by a $100 \mathrm{~cm}^{-1}$ increase in the bend when the (f,d) set is added to the (2d,2p) basis. A third set of first polarization functions has little additional effect. Overall, we conclude there is little net gain in accuracy for calculated vibrational frequencies or geometries at the MP2 level with basis set extension.

The calculations presented above are not absolute "converged," but the similarity of the results for the various correlated calculations in Table 1, coupled with the apparent convergence of the MP2 results as a function of basis, suggests that there are no surprises awaiting if we could extend the theoretical methods to the complete basis set, full CI limit. Were
TABLE 3: HPC Results in 6.31G** Basis $^{a}$

\begin{tabular}{lcccccc}
\hline \multicolumn{1}{c}{ method } & $\begin{array}{c}E \text { (au) } \\
+379.0\end{array}$ & $\begin{array}{c}R_{\mathrm{HP}} \\
(\AA)\end{array}$ & $\begin{array}{c}R_{\mathrm{CF}} \\
(\AA)\end{array}$ & $\omega_{1}, \omega_{2}$ & $\omega_{3}$ & $\omega_{4}$ \\
\hline RHF & +0.0414 & 1.384 & 1.576 & $646 i$ & 1310 & 2695 \\
MP2 & -0.21745 & 1.414 & 1.636 & 334 & 1047 & 2419 \\
MP3 & -0.21896 & 1.398 & 1.598 & $452 i$ & 1245 & 2537 \\
MP4(SDTQ) & -0.26028 & 1.421 & 1.684 & 197 & 790 & 2338 \\
CCSD & -0.23230 & 1.412 & 1.612 & $393 i$ & 1179 & 2414 \\
CCSD(T) & -0.25217 & 1.421 & 1.629 & $326 i$ & 1108 & 2323
\end{tabular}

${ }^{a}$ Frequencies are harmonic frequencies, in $\mathrm{cm}^{-1}$. Only the $l=2$ components of the $\mathrm{d}$ functions are included.

TABLE 4: HPC Results in 6.311G** Basis $^{a}$

\begin{tabular}{lcccccc}
\hline method & $\begin{array}{c}E(\mathrm{au}) \\
+379.0\end{array}$ & $\begin{array}{c}R_{\mathrm{HP}} \\
(\AA)\end{array}$ & $\begin{array}{c}R_{\mathrm{CP}} \\
(\AA)\end{array}$ & $\omega_{1}, \omega_{2}$ & $\omega_{3}$ & $\omega_{4}$ \\
\hline RHF & 0.01079 & 1.385 & 1.573 & $646 i$ & 1203 & 2606 \\
MP2 & -0.26274 & 1.414 & 1.630 & 344 & 1059 & 2408 \\
MP3 & -0.26338 & 1.398 & 1.593 & $450 i$ & 1254 & 2531 \\
CCSD & -0.27650 & 1.411 & 1.608 & $397 i$ & 1185 & 2416 \\
CCSD(T) & -0.29796 & 1.420 & 1.624 & $328 i$ & 1118 & 2324
\end{tabular}

${ }^{a}$ Frequencies are harmonic frequencies, in $\mathrm{cm}^{-1}$.

one to extrapolate these results to the seemingly similar case of linear HPC, one might conclude that the third set of polarization functions is relatively unimportant for the description of the vibrational frequencies and the geometry and that diffuse functions will have a modest effect on the vibrational frequencies. While the former statement appears to be true, it turns out that for HPC diffuse functions seem to have a larger quantitative effect on the bending frequency at the correlated level.

In Table 3 a series of calculations are presented in the 6-31G** basis for HPC. This basis is only double zeta with one set of polarization functions, but the data presented in Table 4 show that 6-311G** results are quite similar both for geometries and for vibrational frequencies. Results in both basis sets are presented to show that the unusual behavior is not unique to either the double zeta or the triple zeta basis. The most startling result from this series of calculations is the oscillation in the bending frequency from real to imaginary as the order of the perturbation theory increases. The RHF results predict that the linear geometry is unstable with respect to bending, but as one moves up in order on the MP perturbation series the linear geometry is either a minimum or saddle point. The CCSD result, which sums the singles and doubles portion of the perturbation series to infinite order, predicts that the linear geometry is unstable with respect to bending. The inclusion of connected triples within the CCSD(T) model suggests the same result, albeit with somewhat smaller imaginary frequency. It is also important to note that the energy contribution of the triples correction is somewhat larger for HPC (approximately $-0.020 \mathrm{~h}$ for HPC vs $-0.014 \mathrm{~h}$ for HCP in the 6-31G** basis, which is also consistent with a larger $T_{1}$ diagnostic for HPC $(0.041)$ than HCP $(0.017))$. This leads to a small lowering of the energy difference between HCP and HPC, of about $4 \mathrm{kcal} /$ mol, when comparing CCSD and CCSD(T) (see Table 5).

Due to the quantitative similarity of the $6-31 \mathrm{G}^{* *}$ and $6-311 \mathrm{G}^{* *}$ results, we chose to examine the effects of diffuse functions in a $6-31++\mathrm{G}^{* *}$ basis; these results are shown in Table 6 . It is seen that diffuse functions have a relatively large effect on the bending frequency at the MP2 level, but little effect on the two stretching frequencies. The trend is to lower the bending force constant (make more negative) in all methods. This was observed in the larger basis sets at the MP2 level as well, but in those basis sets we did not use diffuse functions in the CCSD calculations. Thus in those cases we will assume 
TABLE 5: $\quad \triangle E(H P C-H C P)$ as a Function of Method and Basis Set $^{a}$

\begin{tabular}{clc}
\hline basis set & method & $\Delta E(\mathrm{kcal} / \mathrm{mol})$ \\
\hline $6-311 \mathrm{G}^{* *}$ & RHF & 93.6 \\
& MP2 & 83.3 \\
& MP3 & 87.2 \\
& CCSD & 83.0 \\
& CCSD(T) & 79.1 \\
6-311++G** & MP2 & 82.8 \\
6-311G(2d,2p) & MP2 & 83.6 \\
6-311G(2df,2pd) & MP2 & 83.0 \\
$6-311 G(3 \mathrm{df}, 3 \mathrm{pd})$ & MP2 & 82.2
\end{tabular}

a $\triangle E$ is defined as the energy of HPC minus that of HCP, each at its own stationary point for the method and basis.

TABLE 6: HPC Results in 6-31 $++\mathrm{G}^{* *}$ Basis $^{a}$

\begin{tabular}{lcccccc}
\hline method & $\begin{array}{c}E(\mathrm{au}) \\
+379.0\end{array}$ & $\begin{array}{c}R_{\mathrm{HP}} \\
(\AA)\end{array}$ & $\begin{array}{c}R_{\mathrm{CP}} \\
(\AA)\end{array}$ & $\omega_{1}, \omega_{2}$ & $\omega_{3}$ & $\omega_{4}$ \\
\hline MP2 & -0.22382 & 1.414 & 1.633 & 271 & 1051 & 2410 \\
MP3 & -0.22450 & 1.398 & 1.596 & $473 i$ & 1247 & 2531 \\
CCSD(T) & -0.25792 & 1.421 & 1.627 & $372 i$ & 1110 & 2323 \\
\multicolumn{7}{l}{ Frequencies are harmonic frequencies, in $\mathrm{cm}^{-1}}$.
\end{tabular}

TABLE 7: CCSD Results for HPC vs Basis Set ${ }^{a}$

\begin{tabular}{lcccccc}
\hline \multicolumn{1}{c}{ basis } & $\begin{array}{c}E(\mathrm{au}) \\
+379.0\end{array}$ & $\begin{array}{c}R_{\mathrm{HP}} \\
(\AA)\end{array}$ & $\begin{array}{c}R_{\mathrm{CP}} \\
(\AA)\end{array}$ & $\omega_{1}, \omega_{2}$ & $\omega_{3}$ & $\omega_{4}$ \\
\hline $6-311 \mathrm{G}^{* *}$ & -0.2765 & 1.411 & 1.608 & $397 i$ & 1185 & 2416 \\
$6-311 \mathrm{G}(2 \mathrm{~d}, 2 \mathrm{p})$ & -0.2952 & 1.413 & 1.610 & $469 i$ & 1153 & 2339 \\
$6-311 \mathrm{G}(2 \mathrm{df}, 2 \mathrm{pd})$ & -0.3243 & 1.412 & 1.605 & $436 i$ & 1181 & 2397
\end{tabular}

${ }^{a}$ Frequencies are harmonic frequencies, in $\mathrm{cm}^{-1}$.

that the net effect of expanding the basis set to include diffuse functions would be to make the force constant more negative.

For the smaller basis sets the perturbation series is oscillatory for the character of the linear HPC geometry. In order to investigate the effects of extended basis sets we performed MP2 (results not presented) and CCSD calculations. The results of Table 7 show that CCSD in larger basis sets predicts that HPC is not a minimum but is instead a saddle point, unstable with respect to bending. Basis set extensions beyond the $6-311 \mathrm{G}^{* *}$ level lead to little change in the stretching force constants and small changes in the size of the (imaginary) bending frequency. On the basis of the results presented above for the $6-31 \mathrm{G}^{* *}$ and $6-31++G^{* *}$ basis sets, we predict that extension to the $\operatorname{CCSD}(\mathrm{T})$ level would lead to somewhat smaller imaginary frequencies but that inclusion of diffuse functions would tend to increase the magnitude of the imaginary frequency a similar amount, yielding results similar to the CCSD value in a given basis without diffuse functions. On the basis of the MP2 results we also expect that the further extension to the $6-311 \mathrm{G}(3 \mathrm{df}$, 3 pd) basis would not significantly alter the present conclusions.

(b) Multireference-Based Methods. The $T_{1}$ diagnostic for $\mathrm{HPC}$ in the $\operatorname{CCSD}(\mathrm{T})$ wave function suggests the multireference character of the wave function is significant enough to view the results with caution. ${ }^{22}$ In addition, the oscillatory character of the perturbation theory results leads one to ask whether $\operatorname{CCSD}(\mathrm{T})$ is robust enough to accurately describe HPC and predict the curvature of the potential surface near the linear geometry. With these questions in mind we have applied a variety of multireference based methods to HPC near the linear geometry. The force constants are based on rigid bending of the molecule and are obtained from a fit to three angles. While these are clearly not the "exact" force constants for HPC for the given basis and method (since we are not at the true minimum) they should assess the curvature of the potential near enough to the minimum to test the CCSD and CCSD(T) results. The calculations we performed can be separated into two classes.
TABLE 8: Multireference-Based Results in VDZP ANO Basis $^{a}$

\begin{tabular}{lccc}
\hline \multicolumn{1}{c}{ method } & $E($ linear $)$ & $\begin{array}{c}k- \\
(\text { hartrees/deg }\end{array}$ & $\begin{array}{c}\omega \\
\left(\mathrm{cm}^{-1}\right)\end{array}$ \\
\hline CASSCF(6/6) & -379.11377015 & $-8.19 \mathrm{E}-06$ & $339 i$ \\
CASSCF(8/8) & -379.13933934 & $-1.05 \mathrm{E}-05$ & $384 i$ \\
CASSCF(10/10) & -379.15946624 & $-1.15 \mathrm{E}-05$ & $403 i$ \\
CASSCF(6/6)/MRSDCI & -379.29121061 & $-7.31 \mathrm{E}-06$ & $321 i$ \\
CASSCF(6/6)/MRSDCI+Q & -379.31062332 & $-7.45 \mathrm{E}-06$ & $324 i$ \\
CASSCF(6/6)/MRACPF & -379.30829595 & $-7.53 \mathrm{E}-06$ & $325 i$
\end{tabular}

${ }^{a}$ All force constants and frequencies obtained from rigid bending at standard geometry (see text). Energies are in hartrees.

In the first, we used a small ANO basis to compare the various multireference methods. We then use a subset of the methods to examine the effects of basis set extensions.

The first basis set used is an ANO basis ${ }^{34}$ which is essentially valence double zeta with one set of polarization functions (denoted VDZP below) and is $(8 \mathrm{~s} 4 \mathrm{p} / 2 \mathrm{~s} 1 \mathrm{p})$ for $\mathrm{H},(14 \mathrm{~s} 9 \mathrm{p} 4 \mathrm{~d} /$ $3 \mathrm{~s} 2 \mathrm{p} 1 \mathrm{~d})$ for $\mathrm{C}$, and $(17 \mathrm{~s} 12 \mathrm{p} 5 \mathrm{~d} / 4 \mathrm{~s} 3 \mathrm{p} 1 \mathrm{~d})$ for $\mathrm{P}$. The energies for HPC in this basis at the standard linear geometry, the force constants obtained from the quadratic fit to the three angles, and the estimated vibrational frequency (in $\mathrm{cm}^{-1}$ ) are given in Table 8. This is a modest basis, but serves to compare the various methods. It is seen that all methods predict that HPC is unstable with respect to bending, with force constants similar in size to those found using CCSD and CCSD(T). The CASSCF frequencies are all quite similar to those obtained in the CCSD and $\operatorname{CCSD}(\mathrm{T})$ calculations, with a modest increase in magnitude as the CAS is increased in size. Inclusion of further correlation using all single and double excitations relative to all functions in the 6/6 CASSCF wave function (second-order CI or secondorder ACPF) yields the MRSDCI, MRSDCI+Q, and MRACPF results quoted (CASSCF(6/6)/MRSDCI indicates a single and doubles $\mathrm{CI}$ calculation based using the configurations of the 6/6 CASSCF wave function as a reference space). All three methods produce quite similar estimates of the force constant and the vibrational frequency, and while somewhat different from the various CASSCF results, the frequencies are all within $20 \%$ of each other. In the $10 / 10$ CASSCF wave function for the linear geometry the $\sigma$ occupation numbers for the natural orbitals are $1.98,1.97,1.92,0.09,0.01$, and 0.01 , while the $\pi$ occupation numbers are 1.90 and 0.10 (two sets of $\pi$ orbitals). The $6 / 6$ active space corresponds to removing the two $\sigma$ orbitals with highest occupation numbers and the two $\sigma$ orbitals with lowest occupation numbers from the active space. Since the occupation numbers of the $\sigma$ orbitals neglected in the 6/6 CAS are close to 2 and 0 , respectively, it is reasonable to assume that the effects due to correlating the electrons in these orbitals could be treated in the second-order treatment based on the $6 / 6$ CAS.

We tried a variety of other approaches in the VDZP basis as well. We examined the use of only a subset of the CAS functions as reference functions for all valence-electron $\mathrm{CI}$ or $\mathrm{ACPF}$ equations. The reference functions were chosen as those with coefficients greater than 0.05 or 0.025 in the $6 / 6 \mathrm{CASSCF}$ wave function at the linear geometry. It was found that either reference space led to a zeroth-order wave function which increased in energy upon bending (contrary to the full CASSCF result). In addition, the MRSDCI result in this smaller reference space predicted the linear configuration was a minimum, while the MRSDCI+Q and MRACPF results predicted HPC was unstable with respect to bending. Finally, it was found that the bending force constants for the MRSDCI+Q and MRACPF results in the smaller reference space calculations were significantly larger than those obtained with the full CAS as a 
TABLE 9: Multireference-Based Methods in Larger ANO Basis Sets $^{a}$

\begin{tabular}{lccc}
\hline \multicolumn{1}{c}{ basis, method } & $E$ (linear) & $\begin{array}{c}k \text { - } \\
\left.\text { (hartrees/deg }{ }^{2}\right)\end{array}$ & $\begin{array}{c}\omega \\
\left(\mathrm{cm}^{-1}\right)\end{array}$ \\
\hline VTZ2P, CASSCF(6/6) & -379.11875581 & $-7.04 \mathrm{E}-06$ & $315 i$ \\
VTZ2P, CASSCF(10/10) & -379.16498849 & $-1.05 \mathrm{E}-05$ & $384 i$ \\
VTZ2P, CASSCF(10/12) & -379.19532402 & $-8.34 \mathrm{E}-06$ & $342 i$ \\
VTZ2P, CASSCF(6/6)/ & -379.31609167 & $-6.36 \mathrm{E}-06$ & $299 i$ \\
$\quad$ MRSDCI & -379.33782227 & $-6.67 \mathrm{E}-06$ & $306 i$ \\
VTZ2P, CASSCF(6/6)/ & & & \\
MRSDCI+Q & -379.12296285 & $-8.80 \mathrm{E}-06$ & $352 i$ \\
VQZ3P+(FD), CASSCF(6/6) & -
\end{tabular}

${ }^{a}$ All force constants and frequencies obtained from rigid bending at standard geometry (see text). Energies are in hartrees.

reference; thus we have not considered using the truncated reference space in the larger basis sets.

We were unable to perform second-order CIs based on the $10 / 10$ CASSCF due to the low symmetry upon bending and the large number or configurations generated. However, in order to estimate the effects of reference space expansion in further correlated calculations we examined use of the recently developed CASPT 2 method. The CASPT2 method $^{39}$ is a secondorder perturbation theory based on a CASSCF wave function as a zeroth-order wave function. The zeroth-order Hamiltonian is a sum of Fock-like operators, and the theory reduces to MP2 in the limit that the zeroth-order wave function is a single configuration. We used the form of the theory based on the full nondiagonal Fock operator. We found that the nondiagonal form predicted HPC was unstable with respect to bending for both CASSCFs $(6 / 6$ and 10/10) but the results were quite nonquadratic and tended to flatten out as the angle increased. We thus have not included quadratic force constants for CASPT2. However, we did find that the multireference secondorder method reverses the prediction of MP2 as to the stability of HPC and that there is no significant alteration in the conclusions obtained from the $6 / 6$ reference space when the larger CAS wave functions are used as reference functions.

In Table 9 we present results in an ANO basis set of the form $\mathrm{H} 3 \mathrm{~s} 2 \mathrm{p}, \mathrm{C} 4 \mathrm{~s} 3 \mathrm{p} 2 \mathrm{~d}, \mathrm{P} 5 \mathrm{~s} 4 \mathrm{p} 2 \mathrm{~d}$ (denoted valence triple zeta plus 2 polarization functions; VTZ2P). Due to the similarity between the MRSDCI+Q and MRACPF results quoted above (and in other tests) we only present MRSDCI $+Q$ results. It is seen that HPC is still unstable in the larger basis set, with force constants of similar size to those obtained previously. Further extension to an ANO basis of form H 3s2p1d, C 5s4p3d1f, P 6s5p4d1f (denoted VQZ3P+FD) were examined using the $6 / 6$ CASSCF wave function; these results are also presented in Table 9. It is seen that little change occurs upon basis set extension, relative to the $T Z 2 P$ basis.

\section{Discussion}

The questions we set out to address in the present work were (1) "Is there a stable minimum for HPC at the linear geometry?", and (2) "What is the qualitative nature of the wave function for linear HPC?" We now address them in light of the data presented above.

Concerning question 1 , the present results provide strong evidence that linear HPC is not a minimum on the ground-state surface. All of the CCSD and $\operatorname{CCSD}(T)$ results indicate that the bending frequency is imaginary with a magnitude of between 300 and $400 \mathrm{~cm}^{-1}$. However, on the basis of the $T_{1}$ diagnostic tests performed above one predicts that HPC has significantly more multireference character than $\mathrm{HCP}$ at its minimum, and on this basis one may still view the $\operatorname{CCSD}(\mathrm{T})$ results with some caution. Using a 10/10 CASSCF wave function in the $6-31 \mathrm{G}^{* *}$ basis, we found that the difference between HCP and HPC lies largely in additional excitation out of a $\sigma$ orbital in HPC (natural orbital occupation 1.91, compared to the lowest $\sigma$ NO occupation of 1.97 in HCP). The $\pi$ natural orbital occupations are actually quite similar in the two cases $(1.90, \mathrm{HPC} ; 1.91, \mathrm{HCP})$. This led us to examine HPC using methods that build in the multireference character of HPC at zeroth-order. These methods uniformly lead to the same conclusion as CCSD(T): linear HPC is not a minimum on the ground-state potential surface. Once one uses a CASSCF wave function as a starting point, even a second-order theory predicts this, and the more extensive correlation treatments support this result quantitatively as well as qualitatively.

Of course, the multireference results are based on fixed bond length bending calculations, at a geometry appropriate to CCSD(T) in the $6-31 \mathrm{G}^{* *}$ basis, and one might wonder to what extent this geometry choice affects the computed force constants and frequencies. In preliminary test calculations we found that $\operatorname{CCSD}(\mathrm{T})$ bending force constants calculated at geometries differing by $0.01 \AA$ in both bond lengths could change by approximately $5 \%$, and thus the values quoted above are not definitive for the given multireference method. However, this also suggests that the qualitative result, that linear HPC is not a minimum, is not seriously called into question by possible geometry variations.

One might wonder why it is that the $\operatorname{CCSD}(T)$ method yields results in good agreement with multireference-based methods in a case where it is apparent from the $T_{1}$ diagnostic that multireference character is important. However, a variety of tests of $\operatorname{CCSD}(\mathrm{T})$ have been made and it appears to be unusually robust in cases at least as demanding as the present. For example, $\operatorname{CCSD}(\mathrm{T})$ results with values of $T_{1}$ in the range of $0.04-0.05$ have shown to yield good total energies, geometries, and/or vibrational frequencies when compared with full CCSDT, full CI, or CASSCF studies. ${ }^{40,41}$ In addition, even when $T_{1}$ does get large (e.g., $T_{1}$ equals $0.08,{ }^{1} \Sigma$ state of BN) the error in vibrational frequency relative to a multireference based treatment ${ }^{42}$ is on the order of $50-60 \mathrm{~cm}^{-1}$. When the $T_{1}$ diagnostic is on the order of 0.04 for the ${ }^{3} \Pi$ state of $\mathrm{BN}$ the error in vibrational frequency is much less, on the order of $15 \mathrm{~cm}^{-1}$. Even if our errors are of the same order, this will have no effect on determining whether HPC is a minimum. Thus, it appears that even relatively significant multireference character (it is significant here, note the difference in second-order perturbation theory predictions in going from MP2 to CASPT2N) can be handled by $\operatorname{CCSD}(T)$, and HPC is another example of the robustness of the method.

In comparison with our previous results which were unable to determine whether linear HPC was a minimum, it appears that the flatness of the HPC surface and the multiple bond between $C$ and $P$ led to a case where it was important to be able to (a) sum portions of the perturbation series to a higher order (go beyond second order for a single-reference treatment), and/or (b) explicitly treat the state using a multiconfigurational zeroth-order state. Only when one of these approaches was used could one obtain a consistent description of HPC.

It is apparent from previous studies ${ }^{12-20}$ and the above results that the HCP/HPC system is fundamentally different from $\mathrm{HCN} /$ $\mathrm{HNC}$ in several respects. A recent series of calculations by Bentley et al. ${ }^{2}$ obtains a $14.7 \mathrm{kcal} / \mathrm{mol}$ energy difference between $\mathrm{HCN}$ (lowest energy conformer) and HNC; our previous ${ }^{12}$ and present results suggest that the difference in energy between HCP and HPC is on the order of $80-85 \mathrm{kcal} / \mathrm{mol}$. The HP bond in HPC is much weaker than the HN bond in HNC, if one assumes that the $\mathrm{HC}$ bonds are of comparable strengths. On the basis of average bond enthalpies ${ }^{43}$ one would expect 
the difference to be on the order of about $16 \mathrm{kcal} / \mathrm{mol}$ for conventional HN and HP single bonds, but this accounts for only about one-quarter of the difference in stabilities of the two less stable structures. Another difference is seen in comparing the $\mathrm{HN}$ and HP stretching frequencies in HNC and HPC. For $\mathrm{HNC}$ the $\mathrm{HN}$ stretch is actually somewhat larger than the HC stretch in HCN, but in HPC the HP stretch has dropped significantly relative to the $\mathrm{HC}$ stretch in $\mathrm{HCP}$. This is consistent with the significantly weaker HP bond as well. Finally, the $\mathrm{CN}$ bond in $\mathrm{HCN}$ and $\mathrm{HNC}$ are within $0.01 \AA$ of one another, whereas the CP bonds in HCP and HPC differ by on the order of $0.06-0.07 \AA$. The associated $C X$ vibrational frequency is also reduced to a greater extent in HPC than in $\mathrm{HNC}$, as would be expected based on the lengthened CP bond. Finally, one sees that experimentally and theoretically, $\mathrm{HNC}$ is predicted to be a stable minimum, whereas HPC has not been isolated or observed experimentally.

One can rationalize the differences in stability between HPC and $\mathrm{HNC}$ based on simple ideas of hybridization, and the nature of the bonding required in the two species. In order to preserve the triple bond character in the $\mathrm{CX}$ moiety, $\mathrm{X}$ is forced to hybridize its $\mathrm{s}$ and $\mathrm{p}_{z}$ orbitals when the HXC species is formed if a triple bond to $\mathrm{C}$ is to be maintained. However, when $\mathrm{HCX}$ is formed, $\mathrm{X}$ need not hybridize the ns pair significantly (although it likely does in the case of $\mathrm{HCN}$ ). Thus one can attribute the large difference between HPC and HNC to the relative ease of hybridization for second-row atoms when compared with third-row atoms. Of course, this qualitative picture still does not allow one to predict whether HPC is a stable species or not, except in the limit of an HP bond strength of zero. However, on the basis of the above results it is seen that $\mathrm{HPC}$ is, in fact, not a minimum on the ground-state potential.

It is of interest to note that our present conclusions are consistent with the results of Lehmann et al. ${ }^{7}$ and support our previous CASSCF conclusions. ${ }^{12}$ The unusual harmonicity of the HCP bending potential remains to be treated at high levels, but the present work suggests that $\operatorname{CCSD}(\mathrm{T})$ wave functions will be the minimum level of single-reference correlation treatment adequate to the task. The $(\mathrm{T})$ correction will be important as the molecule bends, as one expects large rearrangements in the orbitals upon correlation for the bent system.

\section{Conclusions}

We have performed calculations using a variety of wave functions to examine the stability of HPC. For single-referencebased methods, depending on the order of the perturbation series, it was found that the MP perturbation series predicts HPC is either a minimum or a saddle point. $\operatorname{CCSD}$ and $\operatorname{CCSD}(\mathrm{T})$ predict HPC is a saddle point. Rigid bending force constants obtained using several multireference correlation treatments support the CCSD and CCSD(T) results. Extensions to the basis sets alter the frequencies to some extent but do not alter this conclusion in a qualitative way.

Acknowledgment. Partial support of this work was provided by the National Science Foundation, grant no. CHE-9222822. R.J.C. also acknowledges the support of the Camille and Henry Dreyfus Foundation through a Camille and Henry Dreyfus Teacher Scholar Award, 1993-98.

\section{References and Notes}

(1) Bowman, J. M.; Gazdy, B.; Bentley, J. A.; Lee, T. J.; Dateo, C. E. J. Chem. Phys. 1993, 99, 308

(2) Bentley, J. A.; Bowman, J. M.; Gazdy, B.; Lee, T. J.; Dateo, C. E. Chem. Phys. Lett. 1992, 198, 563.
(3) Bačić, Z,; Light, J, C. J. Chem. Phys, 1987, 86, 3065.

(4) Bentley, J. A.; Brunet, J.-P.; Wyatt, R. E.; Friesner, R. A.; Leforestier, C. Chem. Phys. Lett. 1989, 161, 393.

(5) Smith, A.; Jørgensen, U. G.; Lehmann, K. K. J. Chem. Phys. 1987, 87,5649

(6) Yang, X.; Rogaski, C. A.; Wodtke, A. M. J. Chem. Phys. 1990 , $92,2111$.

(7) (a) Lehmann, K. K.; Ross, S. C.; Lohr, L. L. J. Chem. Phys. 1985, 82, 4460. (b) Mason, M. A.; Lehmann, K. K. J. Chem. Phys. 1993, 98 , 5184. (c) Lohr, L. L. J. Mol. Spectrosc. 1993, I62, 300.

(8) Chen, Y. T.; Watt, D. M.; Field, R. W.; Lehmann, K. K. J. Chem. Phys. 1990, 93, 2149.

(9) Cabana, A.; Doucet, Y.; Garneau, J.-M.; Pépin, C.; Puget, P. J. Mol. Spectrosc. 1982, 96, 342.

(10) Johns, J. W. C.; Shurvell, H. F.; Tyler, J. K. Can. J. Phys. 1969, 47,893

(11) Tyler, J. K. J. Chem. Phys. 1964, 40, 1170.

(12) Goldstein, E.; Jin, S.; Carillo, M. R.; Cave, R. J. J. Comput. Chem 1993, 14, 186.

(13) Carlsen, N. R. Chem. Phys. Lett. 1977, 47, 203.

(14) Robert, J.-B.; Marsmann, H.; Absar, I.; Van Wazer, J. R. J. Am. Chem. Soc. 1971, 93, 3320.

(15) Thomson, C. Theor. Chim. Acta (Berlin) 1974, 35, 237

(16) Botschwina, P.; Pecul, K.; Preuss, H. Z. Naturforsch. 1975, A30, 1015.

(17) Collins, J. B.; Schleyer, P. v. R.; Binkley, J. S.; Pople, J. A. J. Chem. Phys. 1976, 64, 5142 .

(18) Thomson, C.; Ellam, P. Theor. Chim. Acta (Berlin) 1982, 62, 81.

(19) Karna, S. P.; Bruna, P. J.; Grein, F. Can. J. Phys. 1990, 68, 499.

(20) Bloor, J. E.; Yu, J. J. Phys. Chem. 1990, 94, 5586.

(21) Raghavachari, K.; Trucks, G. W.; Pople, J. A.; Head-Gordon, M. Chem. Phys. Lett. 1989, 157, 479.

(22) Lee, T. L.; Taylor, P. R. Int. J. Quantum Chem. Symp. 1989, 23 199.

(23) Gaussian 92, Revision A. Frisch. M. J.; Trucks, G. W.; HeadGordon, M.; Gill, P. M. W.; Wong, M. W.; Foresman, J. B.; Johnson, B. G.; Schlegel, H. B.; Robb, M. A.; Replogle, E. S.; Gomperts, R.; Andres, J. L.; Raghavachari, K.; Binkley, J. S.; Gonzalez, C.; Martin, R. L.; Fox, D. J.; DeFrees, D. J.; Baker, J.; Stewart, J. J. P.; Pople, J. A. Gaussian Inc., Pittsburgh, PA, 1992.

(24) PSI is an $a b$ initio electronic structure suite for the calculation of SCF, CISD, and CCSD energies, gradients, and vibrational frequencies, written by past and present members of the group of H. F. Schaefer III.

(25) Gdanitz, R. J.; Ahlrichs, R. Chem. Phys. Lett. 1988, 143, 413.

(26) MOLCAS version 2. Andersson, K.; Fülscher, M. P.; Lindh, R.; Malmqvist, P.-A.; Olsen, J.; Roos, B. O.; Sadlej, A. J. University of Lund, Sweden, and Widmark, P.-O. IBM, Sweden, 1991.

(27) Herzberg, G. Molecular Spectra and Molecular Structure: II. Infrared and Raman spectra of Polyatomic Molecules; Krieger: Malabar, FL, 1991; p 173

(28) Langhoff, S. R.; Davidson, E. R. Int. J. Quantum Chem. 1974, 8 , 61.

(29) (a) Hehre, W. J.; Ditchfield, R.; Pople, J. A. J. Chem. Phys. 1972 , 56, 2257. (b) Hariharan, P. C.; Pople, J. A. Theor. Chim. Acta 1973, 28, 213. (c) Gordon, M. S. Chem. Phys. Lett. 1980, 76, 163.

(30) Krishnan, R.; Binkley, J. S.; Seeger, R.; Pople, J. A. J. Chem. Phys. $1980,72,650$.

(31) McLean, A. D.; Chandler, G. S. J. Chem. Phys. 1980, 72, 5639.

(32) Clark, T.; Chandrasekhar, J.; Spitznagel, G. W.; Schleyer, P. v. R. J. Comput. Chem. 1983, 4, 294.

(33) Frisch, M. J.; Pople, J. A.; Binkley, J. S. J. Chem. Phys. 1984, 80 3265.

(34) (a) Widmark, P.-O.; Malmqvist, P. Å.; Roos, B. O. Theor. Chim. Acta 1990, 77, 291. (b) Widmark, P.-O.; Persson, B. J.; Roos, B. O. Theor. Chim. Acta 1991, 79, 419.

(35) GAMESS is a general purpose electronic structure program, originally prepared by M. Dupuis, D. Spangler, and J. J. Wendoloski, at the National Resource for Computations in Chemistry, Software Catalogue, University of California, Berkeley, CA (1980), Program QGO1. The version used in the present work is described in the Quantum Chemistry Program Newsletter: Schmidt, M. W.; Baldridge, K. K.; Boatz, J. A.; Jensen, J. H.; Koseki, S.; Gordon, M. S.; Nguyen, K. A.; Windus, T. L.; Elbert, S. T. QCPE Bull. 1990, 10,52 .

(36) Martin, J. M. L.; Lee, T. J. J. Chem. Phys. 1993, 98, 7951

(37) Pulay, P.; Lee, J.-G.; Boggs, J. E. J. Chem. Phys. 1983, 79, 3382.

(38) Thomas, J. R.; DeLeeuw, B. J.; Vacek, G.; Crawford, T. D. Yamaguchi, Y.; Schaefer, H. H. III. J. Chem. Phys. 1993, 99, 403.

(39) Andersson, K.; Malmqvist, P.-A.; Roos, B. O. J. Chem. Phys. 1992 $96,1218$.

(40) Scuseria, G. E.; Lee, T. J. J. Chem. Phys. 1990, 93, 5851.

(41) Martin, J. M. L.; Lee, T. J. J. Chem. Phys. 1993, 98, 7951

(42) Martin, J. M. L.; Lee, T. J.; Scuseria, G. E.; Taylor, P. R. J. Chem Phys. 1992, 97, 6549 .

(43) Klotz, I. M.; Rosenberg, R. M. Chemical Thermodynamics, 4th ed. Benjamin Cummings: Reading, MA, 1986. 